\title{
Collisionless expansion of pulsed rf plasmas
}

\author{
T Schröder ${ }^{1,2}$, O Grulke ${ }^{1,2}$, T Klinger ${ }^{1,2}, \mathbf{R}$ W Boswell ${ }^{3}$ and C \\ Charles $^{3}$ \\ 1 Max-Planck-Institute for Plasma Physics, EURATOM Association, Greifswald, \\ Germany \\ 2 Ernst-Moritz-Arndt-University, Greifswald, Germany \\ 3 Space Plasma, Power and Propulsion Laboratory, Research School of Physics and \\ Engineering, The Australian National University, ACT 0200 Canberra, Australia \\ E-mail: timo.schroeder@ipp.mpg.de
}

\begin{abstract}
This paper presents experimental results of the quasi isothermal expansion of a pulsed $\mathrm{rf}$ argon plasma $\left(P_{\mathrm{rf}} \approx 300 \mathrm{~W}\right)$ into a vacuum like environment. A fast $\left(\tau_{\text {open }}<200 \mu \mathrm{s}\right)$ gas puffing system prevents significant ionization by energetic electron in the expansion region. Thus the expansion is dominantly determined by ion dynamics. The spatio temporal measurement of the ion saturation current indicates a preceding ion front entangled with a strong potential drop. This is in qualitatively good agreement with the predictions by WIDNER et al., and CROw et al.. Additionally, there is evidence for a supersonic ion population formed by acceleration of the background plasma by the by-passing ion front.
\end{abstract}

PACS numbers: 5275Xx, 5259Dk, 5259Fn

Submitted to: J. Phys. D: Appl. Phys. 


\section{Introduction}

The physics of expanding plasmas into vacuum has been under investigation for decades in order to describe the plasma behaviour around supersonic satellites, during coronal mass ejections or super novas. For simplicity, most of the theoretical approaches investigate the temporal evolution of an initially step-like (semiinfinite) ion density profile with the focus on rarefaction waves. In collisionless, hydrodynamic regimes, and particle simulations, these initializations mostly evolve into self-similar density profiles[1-6]. Although most results are qualitatively comparable, they all converge into different physical limits, e.g. the maximum expansion velocities vary on a scale from the electron thermal velocity $v_{\mathrm{e}}[1,2]$ down to the ion sound velocity $c_{\mathrm{i}}[3-5]$. Experiments carried out in thermionic discharges[7, 8], fireballs[9], and laser produced plasmas[1012] yield that the expansion velocities depend strongly on the electron energy distribution[12-16]. It is predicted that the occurrence of high energetic electrons results in enhanced ion acceleration[7, 18]. This work provides spatio-temporal measurements in a pulsed rf argon discharge with a strong neutral density gradient, for realizing plasma expansion into a vacuum like environment. The results are qualitatively compared with predictions by expansion theory.

\section{Experimental setup}

The Experiments have been carried out in the PIGLET device, i.e. a small linear helicon device described in more detail elsewhere[19]. It consists of a $20 \mathrm{~cm}$ long

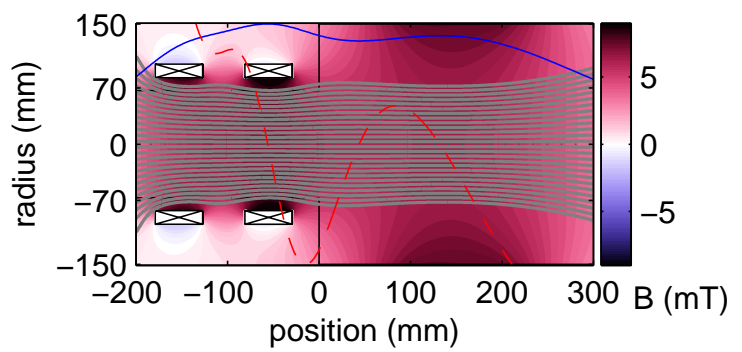

Figure 1: PIGLET device with its magnetic field configuration. The solid blue and the dashed red curve represent the $B_{z}$-profile with $B_{\max }=7.1 \mathrm{mT}$ and the gradient $\nabla B_{z}$ with $\nabla B_{\max }=16.8 \mathrm{mT} / \mathrm{m}$. The magnetic mapping is illustrated as a set of magnetic field lines (grey).

source region attached to a $30 \mathrm{~cm}$ long expansion chamber. The magnetic field coil configuration allows for a homogeneous axial field of approximately $B \approx 6.6 \mathrm{mT}$. The calculated magnetic field configuration is shown in figure 1 . The ripple due to different coil diameters in the source and chamber region is small with $\triangle B / B \leq 15 \%$ and the magnetic field lines are straight. A pulsed rf-generator $(13.56 \mathrm{MHz})$ is used to operate the helicon antenna with a rise time of $\tau \approx 10 \mu \mathrm{s}$. This setup is similar to the one used by Conway et al.[17] to study the ion energy characteristic of a pulsed rf-source. The helicon antenna is impedance matched to provide an optimum coupling during the power rise time up to a peak power of $P_{\mathrm{rf}}=300 \mathrm{~W}$. The fast rise time allows for a localized plasma production in the antenna region, which then can expand into the chamber. Typical plasma parameters and related quantities for the source and chamber region are listed in table. 1. Special attention must be paid to the gas injection system. Firstly, the ignition has to be guaranteed in the 

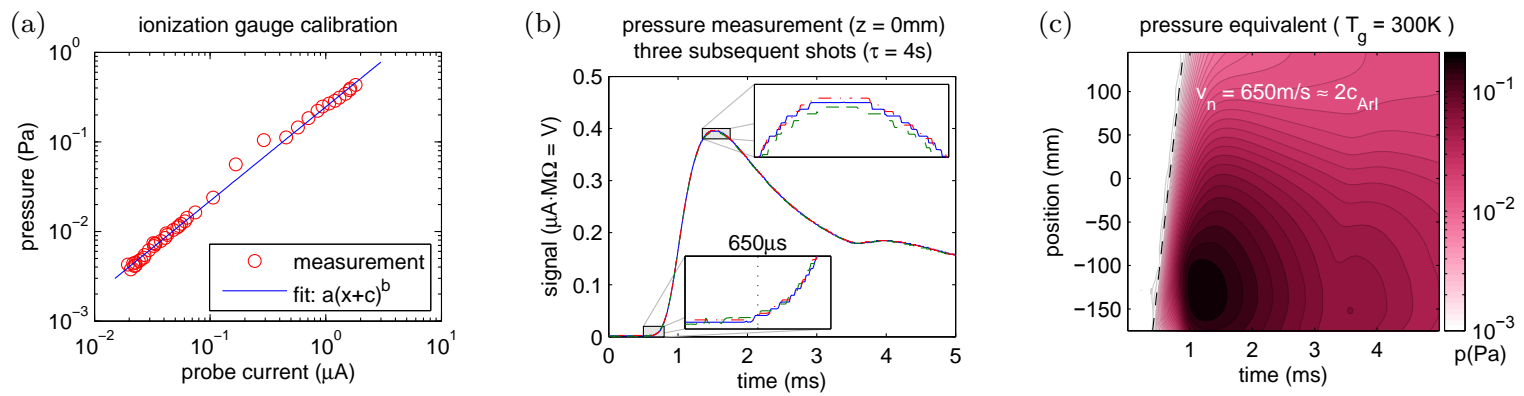

Figure 2: a) Calibration curve of the ionization gauge probe. b) Temporal evolution of the probe signal for three subsequent shots. The time between each shot is $\tau=4 \mathrm{~s}$ to allow for a base pressure below $p_{\mathrm{b}}=10^{-3} \mathrm{~Pa}$. The sub-plots are zoom-ins of the regions marked by the grey rectangle. c) Spatio-temporal measurement of the neutral gas density in absence of a plasma as pressure equivalent for $T_{\mathrm{g}}=300 \mathrm{~K}$. The dashed line marks the gas front velocity $v_{\mathrm{n}}$.

antenna region. Additionally, the neutral gas pressure in the expansion chamber needs to be kept low, to provide a collisionless situation for the ions. This is to prevent a background plasma and setup a situation of plasma expanding into a vacuum like environment.

Thus, a fast solenoid gas valve is installed at the back of the source region at $z_{\mathrm{v}} \approx-195 \mathrm{~mm}$ (fig. 1). The neutral gas density is monitored by a custom made miniaturized hot-filament ionization gauge. The gauge basically consists of three probes: two LANGMUIR probes and one emissive probe. The emissive probe provides electrons constantly drawn by the first LANGMUIR probe biased in the electron saturation regime $\left(U_{\mathrm{e}}=100 \mathrm{~V}\right.$, $\left.I_{\mathrm{e}}=1 \mathrm{~mA}\right)$. The second LANGMuIR probe is in the ion saturation current regime $\left(U_{\mathrm{i}}=-57 \mathrm{~V}\right)$ and its ion current is approximately proportional to the ambient neutral gas density. The probe has been calibrated against a commercial ionization gauge. For the calibration, a needle valve was used providing an adjustable flow of working gas and thus controlling the equilibrium chamber pressure. The calibration curve was proven to be quasi linear over a pressure range of $p_{0}=8$ to $500 \mathrm{mPa}$ (figure $2 \mathrm{a}$ ). The measurement shows the high shot-to-shot reproducibility of the gas puff as shown in figure $2 \mathrm{~b}$. The spatio-temporal evolution of the neutral gas density is depicted in figure 2c. The origin $t_{0}=0$ is given by the TTL-pulse triggering the opening of the valve and the position axis is along the device centre (cf. figure 1). The pulse width defines the time after which the valve current is interrupted allowing for the valve to close again. Prior measurements have shown, that for a pulsewidth of $t_{\mathrm{v}}=130 \mu \mathrm{s}$ a pressure increase is just measurable. Longer pulses increase the throughput of argon until valve is open completely. A further increase of the pulse width would just keep the valve open for longer times and increase the pressure and thus the time to pump down in-between two shots. During the experiment the valve current is interrupted after $t_{\mathrm{v}}=$ $200 \mu \mathrm{s}$. The actual time the valve is open is probably much longer. The closing time of the valve depends on a spring 
and has not been measured directly. One can identify two local density maxima in figure $2 \mathrm{c}$ around $z=-150 \mathrm{~mm}$ : One at $t \approx 1.2 \mathrm{~ms}$ and a smaller one at $t \approx 3.6 \mathrm{~ms}$. The first one is the intended main opening of the valve, whereas the second one is due to a bouncing of the sealing needle when closing. The characteristic closing time by the spring is thus in the order of $1 \mathrm{~ms}$.

By applying a linear fit to the pressure onset (dashed line in figure 2c) one can determine the expansion velocity. The gas expansion is supersonic at a velocity of $v_{\mathrm{n}} \approx 650 \mathrm{~m} / \mathrm{s} \approx 2 \mathrm{c}_{\mathrm{n}}$, where $c_{\mathrm{n}}$ is the sound velocity in neutral argon. This is still much slower than any characteristic plasma velocity. At a time of $t=500 \mu$ s the gas has sufficiently filled the source region for a reliable ignition with a rf-pulse. As can be seen from figure $2 \mathrm{~b}$, the gas puff does not reach the chamber before $t=650 \mu \mathrm{s}$.

The plasma parameters, i.e. plasma potential, electron density, electron temperature, and the ion energy distribution (IED) are measured using an emissive probe, a LANGMUIR probe, a double probe, and a retarding field energy analyser (RFEA), respectively. A brief introduction into RFEAs used for the present study is given by CONWAY et al.[17]. It contains four grids (E, R, D, S) and a collector plate (C) arranged in series. The first grid $\mathrm{E}$ is floating, while the bias of the electrostatic grids $\mathrm{R}$ and $\mathrm{S}$ and the collector $\mathrm{C}$ is set to $-100 \mathrm{~V},-18 \mathrm{~V}$ and $-9 \mathrm{~V}$, respectively. To obtained time resolved measurements of the ion velocity distributions (IVD) the acquisition method is as follows:

At first, the discriminating grid $\mathrm{D}$ of the RFEA is set to a certain voltage $U_{\mathrm{D}}$. The time-resolved collector current $I_{\mathrm{C}}$ is recorded for many pulses to check for repro- ducibility. By repeating this procedure for different voltages $U_{\mathrm{D}}$ one can reconstruct the required $I_{\mathrm{C}}-U_{\mathrm{D}}$-characteristic. Two different profiles have been obtained, one with a RFEA facing up-stream, another facing perpendicular to the magnetic field. Since the perpendicular RFEA collects mainly ions from the bulk plasma, it is used to obtain the plasma potential[19]. The second RFEA is facing up-stream, collecting mostly down-streaming ions. Their drift velocity can be estimated by the difference in the two ion distributions.

In principle, one can obtain the timeresolved $I-U$-characteristic of a LANGMUIR-probe using the method described above. As stated by SEgALL and KoOPMAN[20], LANGMUIR probes should be a reliable diagnostic in streaming plasmas. Nevertheless, the evaluation of the LANGMUIR-probe data remains difficult, since rfcompensation as suggested by SUDIT and CHen is only applicable for high density plasmas[21]. Hence, the LANGMUIR probe is mainly used for the measurement of electron and ion densities using the saturation currents and an estimate for the electron temperature obtained from double probe measurements. This is reasonable since the electron temperature shows only little variation while the densities are expected to vary in the order of magnitudes. The signal noise is of the order $\sigma_{\mathrm{n}}=10^{14} \mathrm{~m}^{-3}$. From this a detection limit has been defined at $10 \sigma_{\mathrm{n}}=10^{15} \mathrm{~m}^{-3}$. All measurements are performed along the central axis of the device.

\section{Simulation}

Expanding plasma is expected to be entangled with the propagation of a strong 
electric field[7]. Thus, the effect of a propagating electric field onto a resting plasma has been studied using a simple single particle simulation. The initial ion energy distribution is a MAXWELLian. The electric field is simulated by a time-dependent amplitude and a spatially GAussian profile. It is determined by:

$E_{0} \propto\left[1+\operatorname{erf}\left(\frac{t}{\sigma_{t}}\right)\right] \exp \left(\frac{\left(x-v_{E} t\right)^{2}}{-2 \sigma_{x}^{2}}\right)$,

where $\sigma_{t}$ defines the smoothness of the onset of the electric field and $\sigma_{x}$ the width of the GAUssian and $v_{E}$ the propagation velocity. A smooth onset can be used to simulate the situation during the formation of the electric field. Time evolution is obtained using a leap-frog method. The electric field perturbation $E_{1}$ can be obtained by successive over-relaxation of the plasma potential under the assumption of the Boltzmann relation.

$E_{1}(x, t) \propto-\frac{\partial \Phi}{\partial x}, \quad \frac{\partial^{2} \Phi}{\partial x^{2}} \propto c \ln (\Phi)-n_{\mathrm{i}}$

The outcome of such a simulation is depicted in figure 3 for three different timesteps.

The electric field $E_{1}$ arising from the density perturbation (2) has two local maxima preceding the original electric field $E_{0}$. The slower of the two arises from ions that where at rest in front of the electric field. As the field passes by they penetrate it and get accelerated until they exit the field region with a velocity twice as fast as the field propagation $\Delta v=2 v_{\mathrm{E}}$.

The second one is due to ions originated near the formation of $E_{0}(1)$ at $x=0$. They were localized close to the upstream edge of the electric field and gain energy by most of the potential drop $\Delta \Phi$. Therefore, they can get much faster $\Delta v=$ $2 v_{\mathrm{E}}+\alpha \Delta \Phi$. Nevertheless, the density of

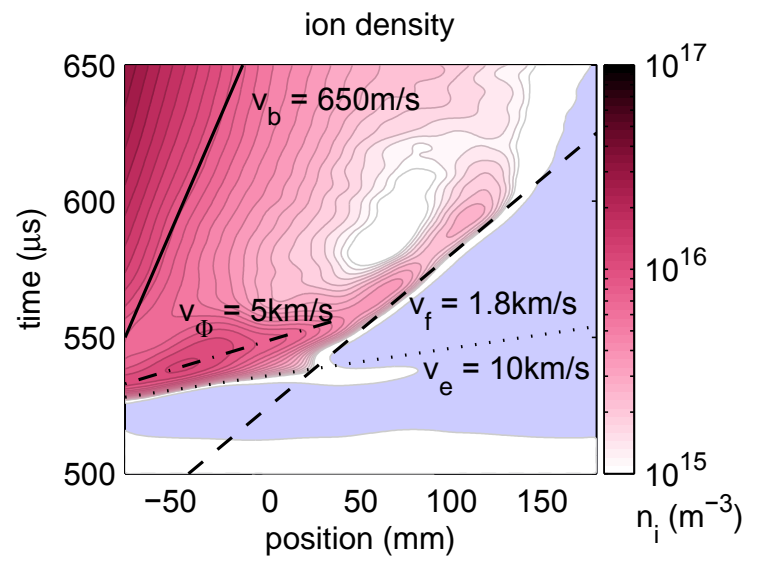

Figure 4: Spatio temporal evolution of the ion density in logarithmic scale. The black lines represent the velocities of the bulk plasma $v_{\mathrm{b}}$ (solid), the isolated ion front $v_{\mathrm{f}}$ (dashed), the potential gradient $v_{\Phi}$ (dashdotted), and the fastest ion population $v_{\mathrm{e}}$ (dotted). The blue area marks the region of very low floating potential allowing for electrons to reach the probe.

the fast front is an order of magnitude lower than the density of the slow one.

\section{Experimental results}

A direct measurement of the electron temperature $T_{\mathrm{e}}$ during the initial phase was not possible. Thus, an estimate is needed to evaluate the probe data. Double probe measurements in cw plasma with comparable parameters yield an electron temperature of $T_{\mathrm{e}} \approx 3$ to $5 \mathrm{eV}$, depending on neutral gas pressure and rf-power. If the temperature variation is small compared to the expected density variation, the influence of electron temperature on the density calculation is negligible, since $I_{\text {sat }_{\mathrm{i}}} \propto n_{\mathrm{i}} \sqrt{T_{\mathrm{e}}}$. Consequently, a constant electron temperature of $T_{\mathrm{e}}=4 \mathrm{eV}$ will be use as first approximation. The ion saturation current can now be used as an 

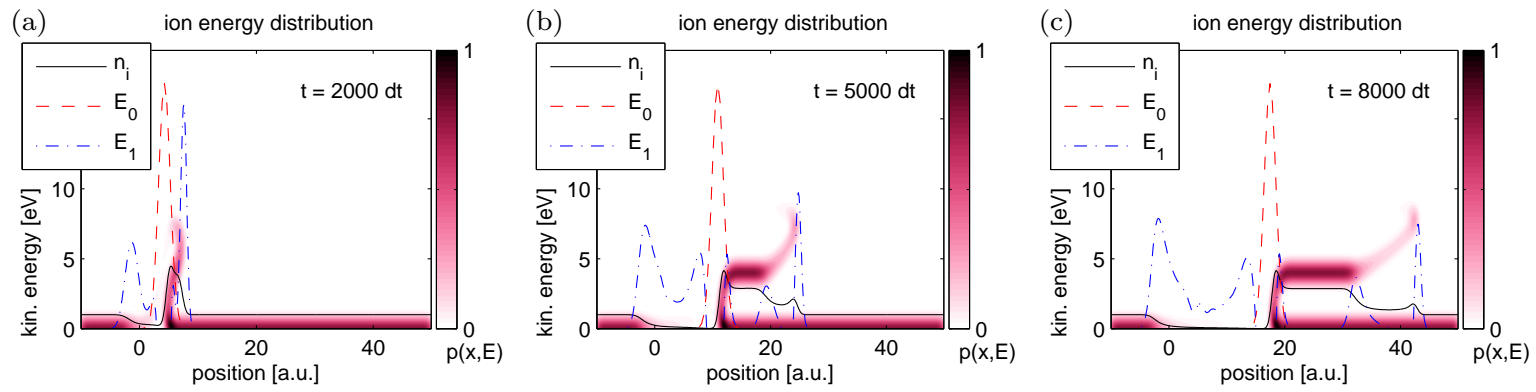

Figure 3: Simulation of a homogeneous background plasma influenced by a bypassing electric field $E_{0}$. Colour coded is the energy distribution function of the background ions $p$, their density distribution $n_{\mathrm{i}}$ and the resulting electric field $E_{1}$ using $n \propto e^{\Phi}$.

(a)

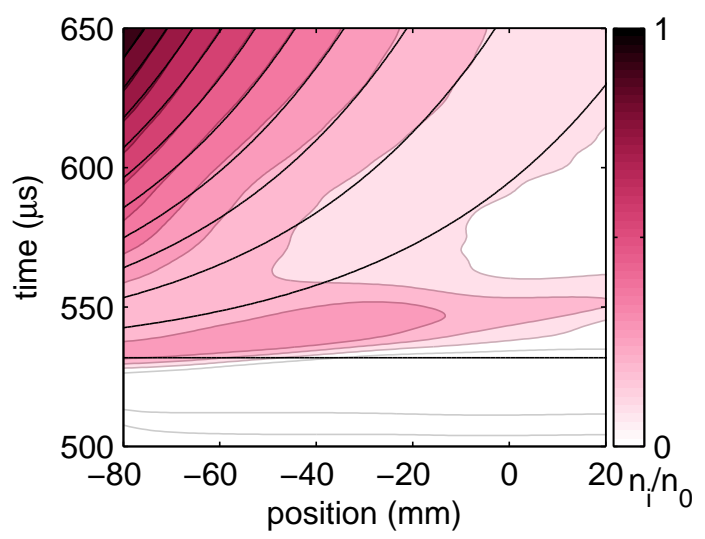

(b)



Figure 5: a) Colour coded spatio temporal profile of the ion density. The fit (3) is given by the dashed contour lines. b) The remaining ion density after subtracting the fit, featuring the pronounced ion front.

approximate measure for the ion density $n_{\mathrm{i}}$ depicted in figure 4 . The spatio-temporal evolution of the bulk plasma density (topleft) is mainly proportional to the evolution of the neutral gas density. As such, it is expanding with the same velocity $v_{\mathrm{b}} \approx$ $650 \mathrm{~m} / \mathrm{s}=\mathrm{v}_{\mathrm{n}}$. Its amplitude is rising linearly in time with a spatial exponential decay. The bulk density can be described well by

$n_{\mathrm{b}}(x, t)=n_{0}\left(t-t_{0}\right) / \tau \exp \left(-\left(x-x_{0}\right) / \lambda\right) \cdot(3)$

The fit to the ion density evolution gives $x_{0} \equiv-80 \mathrm{~mm}, n_{0} \equiv n\left(x_{0}, 650 \mu \mathrm{s}\right), t_{0}=$ $532 \mu \mathrm{s}, \tau=118 \mu \mathrm{s}$, and $\lambda=45.3 \mathrm{~mm}$ (figure 5a) with a relative error of less than $1 \%$. The remaining ion density (figure 5b) can be split into two ion populations, both propagating at much higher velocities. They are launched in the initial phase of the rf-pulse around $(t \approx 525 \mu \mathrm{s})$. The domination population is forming an ion front at a mean velocity of $v_{\Phi} \approx 5 \mathrm{~km} / \mathrm{s}$ in the source region and $v_{\mathrm{f}}=1.8 \mathrm{~km} / \mathrm{s}$ after decelerating in the expansion chamber. This population is much less pronounced if the probe rod is conducting. A second population of tail ions is propagating at a velocity of $v_{\mathrm{e}} \approx$ $10 \mathrm{~km} / \mathrm{s}$. Its density is about an order 


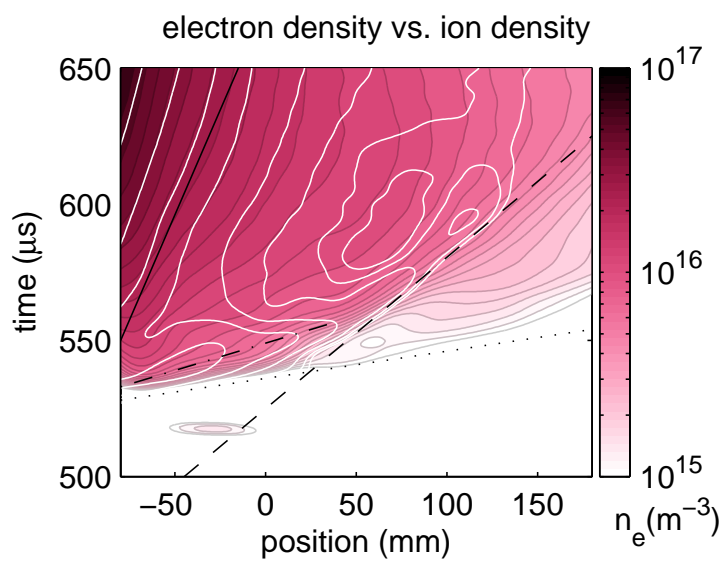

Figure 6: Spatio temporal evolution of the electron density. The ion density is indicated as white contour lines for orientation. The black lines refer to the velocities introduced in figure 4.

of magnitude smaller and drops below the detection limit, soon after exiting the source region (intersection of $v_{\mathrm{f}}$ and $v_{\mathrm{e}}$ ). At times before the ion populations arrive (bottom-right) the ion saturation current is negative. This is due to a floating potential drop caused by energetic electrons. Even a probe bias of $U_{\mathrm{P}}=-90 \mathrm{~V}$ was not sufficient to repel all electrons.

The space-time evolution of the electron density $n_{\mathrm{e}}$ as estimated by the electron saturation current, shown in figure 6 , shows similar features. The fastest electrons are as fast as the tail ion population $v_{\mathrm{e}} \approx 10 \mathrm{~km} / \mathrm{s}$. Further, the electron density is characterized by a drop near the ion front $\left(v_{\mathrm{f}}\right.$ around $\left.t=550 \mu \mathrm{s}\right)$, propagating at the same velocity.

The corresponding potential profile $\Phi$, obtained by the emissive probe, peaks along the trajectory of the ion front (figure 7; white, dashed). Further downstream the plasma potential drops significantly resulting in an electrostatic



Figure 7: Spatio temporal evolution of the plasma potential. The ion density is indicated as black contour lines for orientation. The white lines refer to the velocities introduced in figure 4 .

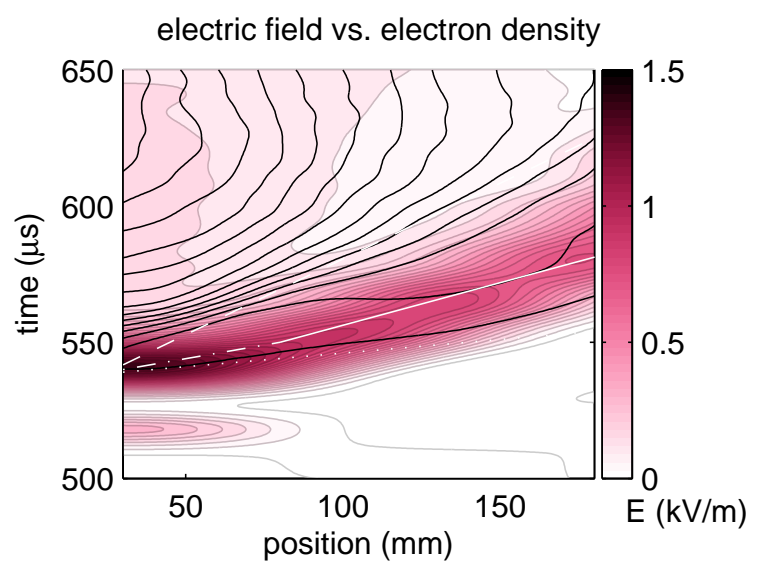

Figure 8: Spatio temporal evolution the derived electrostatic field. The electron density is indicated as black contour lines for orientation. The white lines refer to the velocities introduced in figure 4 . Additionally, the white, solid line marks $v_{\mathrm{e}}=3.2 \mathrm{~km} / \mathrm{s}$.

field. As shown in figure 8 the electric field is very strong at early times close to the source. Its maximum propagates with a velocity of $v_{\Phi}=5 \mathrm{~km} / \mathrm{s}$ at first and than decelerates to $v_{\mathrm{e}}=3.2 \mathrm{~km} / \mathrm{s}$. It is roughly localised between the ion front and the fast 

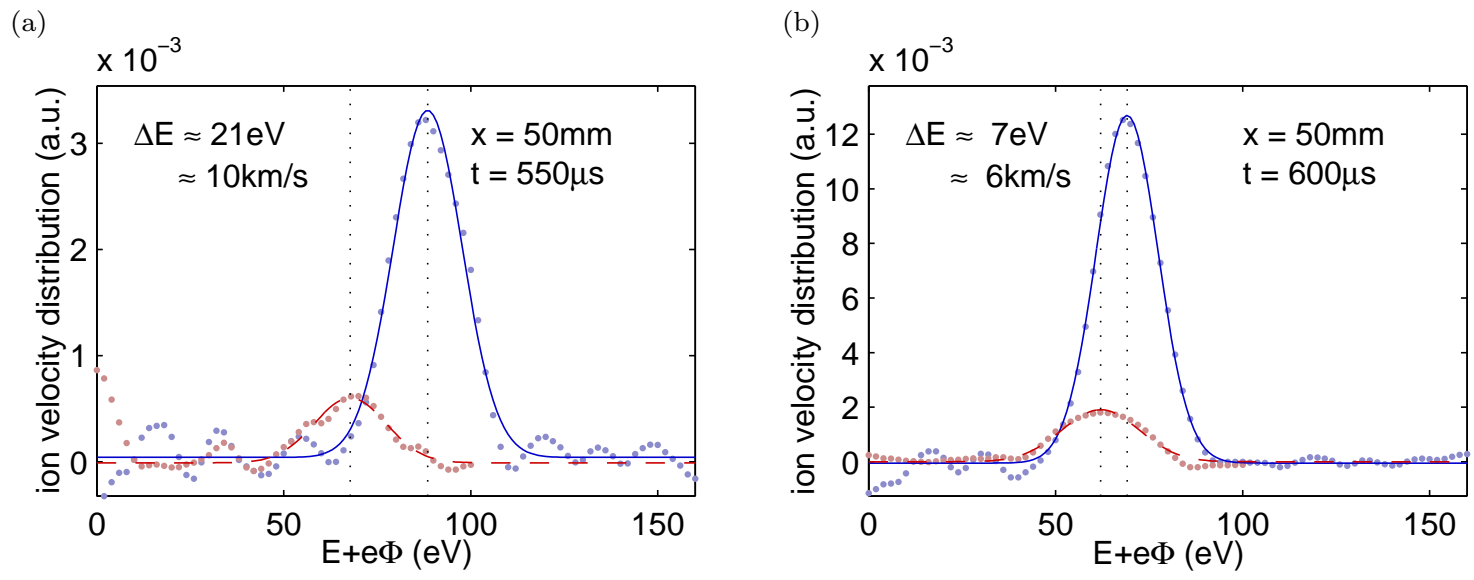

Figure 9: The ion velocity distribution of the ion front (red, solid) in comparison to the ion distribution obtained with a RFEA facing perpendicular to the streaming direction (blue, dashed).

electron population.

The IVDs, depicted in 9a, have been recorded just in front of the ion pronounced front. The downstream IVD (blue, solid) clearly shows an ion population ion around $90 \mathrm{eV}$. With perpendicular IVD data (red, dashed) we obtain a plasma potential of $\Phi \approx 70 \mathrm{eV}$. Taking this as a reference the ion energy of the ion population yields $\Delta E \approx 20 \mathrm{eV}$ which corresponds to a velocity of $v \approx 10 \mathrm{~km} / \mathrm{s}$. The pronounced ion front does not appear in the RFEA data. This is most likely due to the conducting probe rod and body of the RFEA. As mentioned, this behaviour was also observed during the ion saturation measurement. Nonetheless, the IVD at the same position but after the bypass of the front shows a much smaller drift velocity of $v \approx 5$ to $6 \mathrm{~km} / \mathrm{s}$. The plots show the poor resolution of the RFEA leaving an uncertainty of at least $1 \mathrm{eV}$. The RFEA signal collects electrons at the time the LANGMUIR measurement encountered the floating potential drop. Thus, the kinetic energy of the electrons is at least $E_{\mathrm{e}}=$
$100 \mathrm{eV}+\mathrm{e} \Phi$

\section{Discussion}

The experimental results show a preceding supersonic ion front and a continuous expanding electron cloud during the expansion. All together, four characteristic velocities can be identified. All four velocities are imprinted in the spatio-temporal ion density/saturation current evolution. The majority of the plasma is expanding in the same manner as the neutral gas with a velocity of $v_{\mathrm{b}} \approx 2 c_{\mathrm{n}}$. The first detached population is initially propagating with $v_{\Phi}=5 \mathrm{~km} / \mathrm{s}$. After entering the expansion chamber, the population decelerates and the propagation velocity $v_{\mathrm{f}}=1.8 \mathrm{~km} / \mathrm{s}$ becomes subsonic, assum$\operatorname{ing} T_{\mathrm{e}}=3$ to $5 \mathrm{eV}$. From our setup, this seems to be most likely due to the change of radial boundary conditions. Nevertheless, such a deceleration has also been reported by Hairapetian and Stenzel[8], where it was disentangled from any change of boundary conditions. The ions carry 
a significant positive charge resulting in a local maximum of the plasma potential. Beyond the ion front, a high energetic $\left(E_{\mathrm{e}}>100 \mathrm{eV}\right)$ electron population is affecting the RFEA and LANGMUIR probe measurements. This fits well with the existence of an energetic electron tail in the velocity distribution, as pointed out before[18]. In the source region there is evidence of a faster ion population propagating with twice the initial front velocity $v_{\mathrm{e}}=10 \mathrm{~km} / \mathrm{s}$. The evidence gets supported by the electron density evolution and the IVD measurements in front of the ion front. Both indicate the same drift velocity. This second ion population is most likely accelerated by the mechanism described in the simulation.

The space charge of the preceding electrons together with the space charge of the ion front form a propagating electric field. Due to the different drift velocities of the charge layers, the electric field broadens and propagates with an intermediate velocity. The electric field propagates approximately at the ion sound speed.

In principle, the second ion population should form a new ion front resulting in a new electric field that again would accelerate a small amount of ions. The RFEA measurement can not resolve this fine structure as it would be beyond the measuring range $(E>80 \mathrm{eV})$ and secondly the density would be too small compared to the noise level.

Since there are many different theories about plasma expansion mechanisms, in the following the experiment is compared to the tree major systems. HENDEL et al.[10] described adiabatic acceleration to be the most promising candidate for the ion acceleration process during

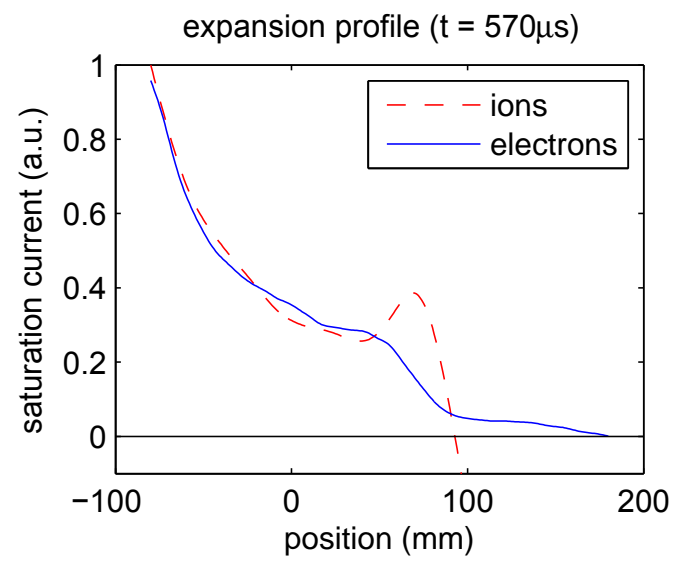

Figure 10: Ion (red, dashed) and electron (blue, solid) saturation current in normalized units.

plasma expansion. But since the rf source continuously provides energy to the system, their calculations for the ion drift velocity do not apply to the present experiments. An isothermal expansion model should be more applicable.

For a planar isothermal expansion of a semi-infinite plasma a numerical investigation has been carried out by Crow et al.[2]. The measured ion and electron saturation currents (figure 10) are in good agreement with their calculated profiles. As pointed out by the authors, the theory predicts a permanently nonzero acceleration of the ion population until the theory looses its validity, i.e. when the ion velocity is of the order of the electron thermal velocity $v_{\mathrm{th}_{\mathrm{e}}}$. However, the observations show much weaker acceleration and maximum ion velocity $v_{\mathrm{e}} \ll v_{\mathrm{th}}$.

Simulations carried out by WIDNER et al. [4] predict an ion burst with a velocity of approximately $3 c_{\mathrm{i}}$. Under this assumption, velocity of $v_{\mathrm{e}} \approx 10 \mathrm{~km} / \mathrm{s}$ requires an electron temperature of $T_{\mathrm{e}}=4.6 \mathrm{eV}$, which 
lies within the experimentally obtained range.

\section{Summary and conclusion}

The experimental results show several features that find their qualitative equivalents in expansion experiments and theory. The majority of the plasma density is contained in the bulk plasma. The bulk plasma expansion is quasi-neutral and its spatial density profile can be described by an exponential. Its evolution follows roughly the neutral gas density evolution as it is mainly formed by local ionization.

In addition to the bulk plasma dynamic, there is a much faster expansion of a small fraction of the plasma. The evolution of the ion density has a distinct isolated ion population as predicted by Widner et al.[4] and Crow et al.[2]. The ion population is preceded by electrons forming a propagating electrostatic double layer (DL). The resulting electric field is carried along with the ions at approximately the ion sound velocity. Such a potential drop has also been reported by Hairapetian and Stenzel[7,8] in thermionic discharges. This indicates that the DL formation is rather independent of the type of source. Even the slow down of the DL is in agreement with their experimental data. Nevertheless, the DL observed in present work is not stopping entirely, but propagating with an ever finite velocity until it reaches the end plate.

As the DL is passing by the downstream plasma that has formed due to the preceding electrons it accelerates the ions to approximately twice its initial velocity, i.e. before deceleration. This fastest ion population reaches velocities of approxi- mately three times the ion sound speed as predicted by Widner et al.[4]. The acceleration process can be described by a simple model of a propagating electric field passing by a resting plasma.

Although the RFEA is not able to directly resolve the ion front, it detects ions with similar velocities after the ion front has passed by. These ions draw their kinetic energy continuously from the spatial plasma potential gradient. Thereby they can gain maximum energies of 20 to $30 \mathrm{eV}$ corresponding to velocities of 10 to $12 \mathrm{~km} / \mathrm{s}$ or $3 c_{\mathrm{i}}$ to $4 c_{\mathrm{i}}$. The data shows that ion acceleration up to electron thermal velocities as predicted by CROw et al.[2] is either not occurring at all or only for a insignificant fraction of ions ( $\ll 1 \%)$. Even the amount of ions that gain velocities in the order of $v_{\mathrm{e}} \approx 3 c_{\mathrm{i}}[4]$ is relatively small $(\approx 1 \%)$. Nonetheless, in astrophysical plasma expansions the absolute quantities of these fast populations can be much higher.

\section{Acknowledgments}

The author would like to thank the DAAD (German Students Exchange Service) for funding and the colleagues of the RSPE, ANU, Canberra for their support and hospitality.

\section{References}

[1] Allen J E and Andrews J G $1970 \mathrm{~J}$. Plasma Phys. 4, 187-\&.

[2] Crow J E, Auer P L and Allen J E 1975 J. Plasma Phys. 14, 65-76.

[3] Gurevich A V, Pariiska I V and Pitaevsk L P 1966 JETP Letters 22, 449-\&. 
[4] Widner M, Alexeff I and Jones W D 1971 Phys. Fluids 14, 795-\&.

[5] Mora P and Pellat R 1979 Phys. Fluids 22, 2300-2304.

[6] Perego M, Howell P D, Gunzburger $\mathrm{M} \mathrm{D}$, Ockendon J R and Allen J E 2013 Phys. Plasmas 20, 052101.

[7] Hairapetian G and Stenzel R L 1988 Phys. Rev. Lett. 61, 1607-1610.

[8] Hairapetian G and Stenzel R L 1991 Phys. Fluids B 3, 899-914.

[9] Stenzel R 2008 Plasma Sources Sci. Technol. 17, 035006.

[10] Hendel H W and Reboul T T 1962 Phys. Fluids 5, 360-362.

[11] Kroesen G M W, Schram D C, Wilbers A T M and Meeusen G J 1991 Contrib. Plasma Phys. 31, 27-42.

[12] Gurevich A V and Meshcherkin A P 1981 JETP Letters 80, 1810-1826.

[13] Bezzerides B, Forslund D W and Lindman E L 1978 Phys. Fluids 21, 2179-2185.

[14] Pearlman J S and Morse R L 1978 Phys. Rev. Lett. 40, 1652-1655.

[15] Denavit J 1979 Phys. Fluids 22, 13841392.

[16] Mordvinov A V, Tomozov V M and Fainshtein V G 1985 J. Appl. Mech. Tech. Phys. 26, 764-8.

[17] Conway G D, Perry A J and Boswell R W 1998 Plasma Sources Sci. Technol. 7, 337-347.

[18] True M A, Albritton J R and Williams E A 1981 Phys. Fluids 24, 1885-1893.

[19] Lafleur T 2009 Phys. Plasmas 16, 044510 .

[20] Segall S B and Koopman D W 1973 Phys. Fluids 16, 1149-1156.
[21] Sudit I D and Chen F F 1994 Plasma Sources Sci. Technol. 3, 162-168.

\begin{tabular}{|c|c|c|c|}
\hline & & char & un \\
\hline neutral pressure: & $10^{-1}$ & $10^{-3}$ & $\overline{\mathrm{Pa}}$ \\
\hline peak rf power: & \multicolumn{2}{|c|}{300} & W \\
\hline avg. magnetic field: & \multicolumn{2}{|c|}{6.6} & $\mathrm{mT}$ \\
\hline electron density: & $10^{16}$ & $10^{15}$ & $\mathrm{~m}^{-3}$ \\
\hline electron temperature: & \multicolumn{2}{|c|}{$3-5$} & $\mathrm{eV}$ \\
\hline ion temperature: & \multicolumn{2}{|c|}{0.026} & $\mathrm{eV}$ \\
\hline plasma frequency: & $5.6 \cdot 10^{9}$ & $1.8 \cdot 10^{9}$ & $\mathrm{~s}^{-1}$ \\
\hline ion plasma freo & $2.1 \cdot 10^{7}$ & $6.6 \cdot 10^{6}$ & $\mathrm{~s}^{-1}$ \\
\hline electron thermal velocity: & \multirow{2}{*}{\multicolumn{2}{|c|}{$\begin{array}{c}1.0-1.3 \cdot 10^{6} \\
3.5 \cdot 10^{2}\end{array}$}} & $\mathrm{~m} / \mathrm{s}$ \\
\hline ion thermal velocity: & & & $\mathrm{m} / \mathrm{s}$ \\
\hline ion sound speed: & \multicolumn{2}{|c|}{$2.7-3.5 \cdot 10^{3}$} & $\mathrm{~m} / \mathrm{s}$ \\
\hline Debye length: & $1.5 \cdot 10^{-4}$ & $5.0 \cdot 10^{-4}$ & $\mathrm{~m}$ \\
\hline electron mean free path: & $2.5 \cdot 10^{-1}$ & $4.1 \cdot 10^{0}$ & $\mathrm{~m}$ \\
\hline ion mean free path: & $1.1 \cdot 10^{-3}$ & $1.3 \cdot 10^{-2}$ & $\mathrm{~m}$ \\
\hline electron-electron collision: & $2.4 \cdot 10^{6}$ & $2.6 \cdot 10^{5}$ & $\mathrm{~Hz}$ \\
\hline electron-neutral collision: & $2.3 \cdot 10^{6}$ & $2.3 \cdot 10^{4}$ & $\mathrm{~Hz}$ \\
\hline electron-ion collision: & $5.1 \cdot 10^{4}$ & $5.5 \cdot 10^{3}$ & $\mathrm{~Hz}$ \\
\hline ion-ion collision: & $2.5 \cdot 10^{5}$ & $2.8 \cdot 10^{4}$ & $\mathrm{~Hz}$ \\
\hline ion-neutral collision: & $3.6 \cdot 10^{3}$ & $3.6 \cdot 10^{1}$ & $\mathrm{~Hz}$ \\
\hline charge exchange collision: & $6.4 \cdot 10^{4}$ & $6.4 \cdot 10^{2}$ & $\mathrm{~Hz}$ \\
\hline
\end{tabular}

Table 1: List of plasma parameter and related quantities. 